\title{
Single-walled carbon nanotube-induced crystallinity on the electropolymeric film of tetraaminophthalocyaninatonickel(II) complex: Impact on the rate of heterogeneous electron transfer
}

\author{
Jeseelan Pillay $^{a}$ and Kenneth I. Ozoemena ${ }^{a}$ \\ ${ }^{a}$ Chemistry Department, University of Pretoria, Pretorial 0002, South Africa
}

\begin{abstract}
We present a fundamental investigation on the impact of single-walled carbon nanotube (SWCNT) on the film structure and redox behaviour of tetraaminophthalocyaninatonickel(II) complex (NiTAPc) electropolymer immobilized on a basal plane pyrolytic graphite electrode (BPPGE). SWCNT induces crystallinity on the NiTAPc electropolymeric film structure and increases its apparent electron transfer rate constant $\left(k_{\text {app }}\right)$. We proved that there is potential advantage of using electrode based on the SWCNT-poly-NiTAPc hybrid for catalytic and sensing applications as it enhances the catalytic current for the detection of nitric oxide more than twice compared to bare BPPGE, BPPGE-SWCNT and other electrodes without SWCNTs.
\end{abstract}

\section{Graphical abstract}

Single-walled carbon nanotubes induce crystallinity on NiTAPc electropolymer, affect the redox properties of NiTAPc film and enhances its ability to detect nitric oxide. 


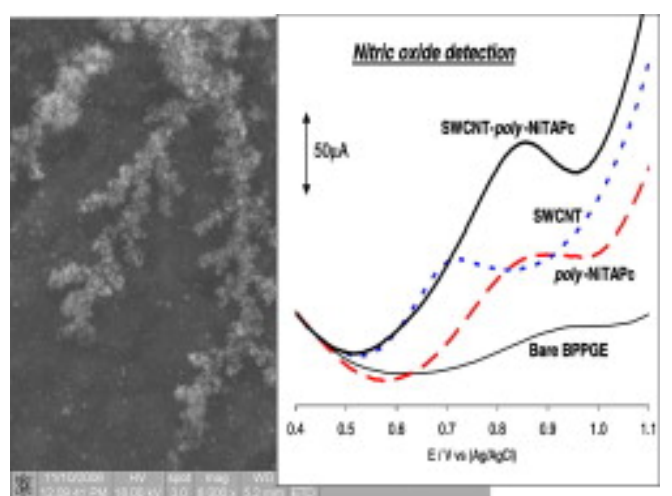

\section{Article Outline}

1. Introduction

2. Experimental

2.1. Materials and reagents

2.2. Apparatus and procedure

2.3. Electrode modification and pretreatments

3. Results and discussion

3.1. FESEM characterization of the BPPGE-SWCNT-poly-NiTAPc

3.2. Impact of scan number on the electron transport

3.3. Comparative electron transport properties

3.4. Comparative film capacitances

3.5. Comparative electrocatalytic behaviour towards nitric oxide

4. Conclusion

Acknowledgements

References

\section{Introduction}

The excellent physico-chemical [1] and electrocatalytic features [2] and [3] of carbon nanotubes (CNTs) are well established. The remarkable redox chemistries of metallophthalocyanine (MPc) complexes have also been well documented [4], [5] and 
[6]. It is now known that carbon nanotubes enhance the electrocatalytic and electronic properties of phthalocyanine and related porphyrin complexes [7], [8], [9], [10] and [11]. One of the many intriguing properties of CNTs is their ability to induce crystallinity on polymers prepared by solution or melt-processing techniques [12], [13], [14], [15], [16], [17], [18] and [19]. To our knowledge, the induction of crystallinity by CNTs on polymers prepared by electrochemical techniques has not been reported, and we are not aware of any such observation for the electropolymerized films of metallophthalocyanine (MPc). As reviewed by Batail et al. [20], the importance of electrocrystallization as an invaluable tool for the construction of ordered electroactive molecular solids cannot be over emphasized. Electrochemical synthesis of redox-active crystals on electrode surface may be exploited for the preparation of high-performance polycrystalline electrodes. Electrodeposition offers high degree of freedom to control the amount of deposits, and may lead to creation of nanocrystalline electrodes of not only high surface areas but also superior charge transport properties to those of individual particles [21]. In this work, we report the first observed SWCNT-induced microcrystallinity on the electropolymer of phthalocyaninatonickel (II) complex (NiTAPc) on BPPGE (via field emission scanning electron microscopy) and the electron transfer kinetics of the electropolymer (via cyclic voltammetry and impedance spectroscopy) of the SWCNT and electropolymeric films of NiTAPc on BPPGE. Of all the known MPc complexes, electropolymeric films of nickel phthalocyanine ( $\mathrm{NiPc}$ ) complexes remain the most studied for the electrochemical detection of nitric oxide, an important neurotransmitter [22], [23], [24] and [25]. Thus, a preliminary voltammetric detection for $\mathrm{NO}$ is discussed.

\section{Experimental}

\subsection{Materials and reagents}

Single-walled carbon nanotubes (SWCNTs, Aldrich) was acid-digested using the known procedure [26]. Tetraaminophthalocyaninatonickel(II) (NiTAPc) complex was kindly donated to us by Prof. Nyokong (Rhodes University). Ultra pure water of resistivity 18.2 M $\Omega$ was obtained from a Milli-Q Water System (Millipore Corp., Bedford, MA, USA) and was used throughout for the preparation of solutions. Phosphate buffer solutions (PBS) at various pHs were prepared with appropriate amounts of $\mathrm{K}_{2} \mathrm{HPO}_{4}$ and 
$\mathrm{KH}_{2} \mathrm{PO}_{4}$, and the $\mathrm{pH}$ adjusted with $0.1 \mathrm{M} \mathrm{H}_{3} \mathrm{PO}_{4}$ or $\mathrm{NaOH}$. All electrochemical experiments were performed with nitrogen-saturated PBS. All other reagents were of analytical grades and were used as received from the suppliers without further purification.

\subsection{Apparatus and procedure}

Field emission scanning electron microscopy (FESEM) images were carried out with FEI Nova NanoSEM 200 (Japan) at the AuTEK Nanotechnology Laboratory of MinTEK. Electrochemical data were obtained with Autolab Potentiostat PGSTAT 302 (Eco Chemie, Utrecht, The Netherlands) driven by the GPES software version 4.9. Electrochemical impedance spectroscopy (EIS) measurements were performed with an Autolab Frequency Response Analyser (FRA) software between $1.0 \mathrm{~Hz}$ and $10 \mathrm{kHz}$ using a $5 \mathrm{mV}$ rms sinusoidal modulation with a solution of $1 \mathrm{mM}$ of $\mathrm{K}_{4} \mathrm{Fe}(\mathrm{CN})_{6}$ and $1 \mathrm{mM}$ $\mathrm{K}_{3} \mathrm{Fe}(\mathrm{CN})_{6}(1: 1)$ mixture containing $0.1 \mathrm{M} \mathrm{KCl}$ or PBS solutions of this mixture at different $\mathrm{pHs}$, and at the $E_{1 / 2}$ of the $\left[\mathrm{Fe}(\mathrm{CN})_{6}\right]^{3-/ 4-}(0.124 \mathrm{~V}$ versus $\mathrm{Ag} \mid \mathrm{AgCl})$. Bare or modified basal plane pyrolytic graphite (BPPGE) disk ( $d=4.8 \mathrm{~mm}$ in Teflon) was used as the working electrode and was fabricated in-house at our Chemistry workshop using BPPG plate (Le Carbone, Sussex, UK). Electrical contact with the disk was maintained through an inserted copper wire held in place with conducting silver varnish L 100 (Kemo_Electronic, Germany). A $\mathrm{Ag} \mid \mathrm{AgCl}$ wire and platinum wire were used as pseudoreference and counter electrodes, respectively. A bench top pH/ISE ORION meter, model $420 \mathrm{~A}$, was used for $\mathrm{pH}$ measurements. All solutions were de-aerated by bubbling nitrogen prior to each electrochemical experiment. Experiments were performed at $25 \pm 1{ }^{\circ} \mathrm{C}$.

\subsection{Electrode modification and pretreatments}

First, the BPPGE surface was cleaned by gentle polishing on a p1200C Norton carborundum paper (Saint-Gobain Abrasives, Isando, South Africa). This was followed by cleaning with cellotape to remove graphite layers and finally rinsed in acetone to remove any adhesive on the electrode surface. The NiTAPc modified BPPGE-SWCNT was obtained by immersing a BPPGE-SWCNT (obtained by drop-dry method) in a 
solution of $c a .5 \mathrm{mM}$ NiTAPc in dry DMF containing $10 \mathrm{mM}$ tetrabutylammonium tetrafluroroborate (TBABF4) as the supporting electrolyte and repetitively scanned (5-40 scan range) between $-0.4 \mathrm{~V}$ and $1.2 \mathrm{~V}$ potential window. As will be discussed latter, the electropolymer obtained at 7 scan number (represented herein as BPPGE-SWCNT-poly$\mathrm{NiTAP}_{7}$ ) was used for most of the experiment, unless otherwise stated. Similar procedure was used to obtain the BPPGE-poly-NiTAPc ${ }_{7}$ electrode. The BPPGE-poly$\mathrm{Ni}(\mathrm{OH}) \mathrm{TAPc}_{7}$ was obtained from BPPGE-poly-NiTAPc - $_{7}$ by continuous scanning in a $0.1 \mathrm{M} \mathrm{NaOH}$.

\section{Results and discussion}

\subsection{FESEM characterization of the BPPGE-SWCNT-poly-NiTAPc}

Electropolymerization of the NiTAPc onto a SWCNT modified BPPGE followed the established methods for the formation of NiTAPc polymer on carbon electrodes [23], [24] and [25]. Fig. 1 is a typical FESEM image of the electropolymeric film. 


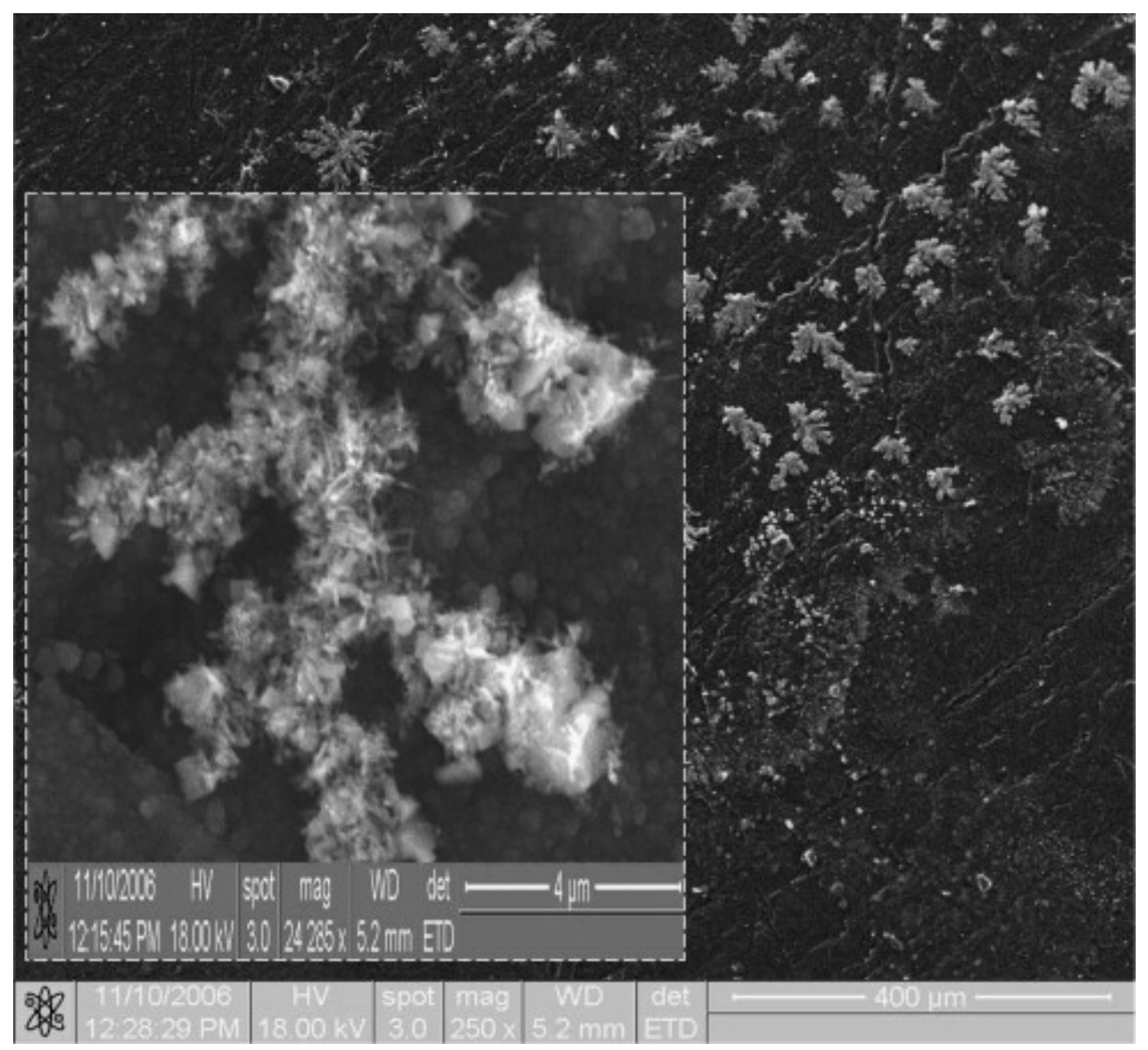

Fig. 1. FESEM image of BPPGE-SWCNT-NiTAPc at 250× magnification. Inset shows the crystalline structure of BPPGE-SWCNT-NiTAPc at increased (24 285×) magnification. Conditions: electropolymeric film was obtained by repetitive scanning ( 7 scans) of a $5 \mathrm{mM}$ NiTAPc in DMF containing $\mathrm{TBABF}_{4}$ at a BPPGE-SWCNT. The electropolymer was also successfully transformed into the $\mathrm{O}-\mathrm{Ni}-\mathrm{O}$ oxo-bridged derivatives [23], [24] and [25], BPPGE-SWCNT-poly-Ni(OH)TAPc, unfortunately, unlike the BPPGE-SWCNT-poly-NiTAPc, the BPPGE-SWCNT-poly-Ni(OH)TAPc peels off from the electrode in aqueous solution, so no further experiment was carried out with this electrode.

It is seen from the high FESEM magnification (Fig. 1, inset) that the BPPGE-SWCNTpoly-NiTAPc 7 gave a dendrite-like microcrystal structure, which we attribute to the presence of the SWCNT. At the low magnification (Fig. 1), it is clear that the BPPGE is not completely covered by these SWCNT-NiTAPc crystals, but are randomly distributed 
throughout the electrode surface. This behaviour is attributed to the fabrication method; drop-coating of SWCNT with the accompanying mild oven-drying encourages aggregation and breaking of the immobilized nanotubes into microscopic pieces. The obtained crystalline NiTAPc electropolymers are concentrated on these microscopic pieces of the immobilized SWCNTs, further confirming the ability of the SWCNT to induce crystallinity on the NiTAPc electropolymer. Although, it has been widely reported that CNTs are capable of inducing crystallinity on polymeric species [11], [12], [13], [14], [15], [16], [17], [18] and [19], this represents the first report of such observation for an electropolymer and especially for an MPc electropolymer.

\subsection{Impact of scan number on the electron transport}

Impedance spectroscopy provides an excellent means of probing the electron transfer properties of modified electrodes [27] and [28]. Crystalline structures are efficient intermedia for interchain transport of charge carriers [21], thus we examined the electron transport features of the films using a simple electrochemically reversible redox probe, $\left[\mathrm{Fe}(\mathrm{CN})_{6}\right]^{3-/ 4-}$. Fig. 2 presents the plots of the peak-to-peak separation $\left(\Delta E_{\mathrm{p}}\right)$ and the apparent electron transfer constants $\left(k_{\text {app }}\right)$ versus the scan number, indicating the influence of electropolymerization scan numbers on the electron transport of the BPPGESWCNT-poly-NiTAPc. The $k_{\text {app }}$ values were extracted from the electron transfer resistance $R_{\mathrm{CT}}(\Omega)$ values obtained from the semi-circle of the Nyquist plots using the following equation:

$$
h_{\text {का }}=\frac{R T}{H^{2} F^{2} A B_{C T} C}
$$

where $n$ is the number of electron transferred (1), $A$ is the geometric area of the electrode $\left(0.181 \mathrm{~cm}^{2}\right), C$ is the concentration of the $\left[\mathrm{Fe}(\mathrm{CN})_{6}\right]^{3-}$ (in mol cm${ }^{-3}$, the concentration of $\left[\mathrm{Fe}(\mathrm{CN})_{6}\right]^{3-}$ and $\left[\mathrm{Fe}(\mathrm{CN})_{6}\right]^{4-}$ are equal), $R, T$ and $F$ have their usual meanings. The plots show that the CV results agree qualitatively well with the observed EIS data. 


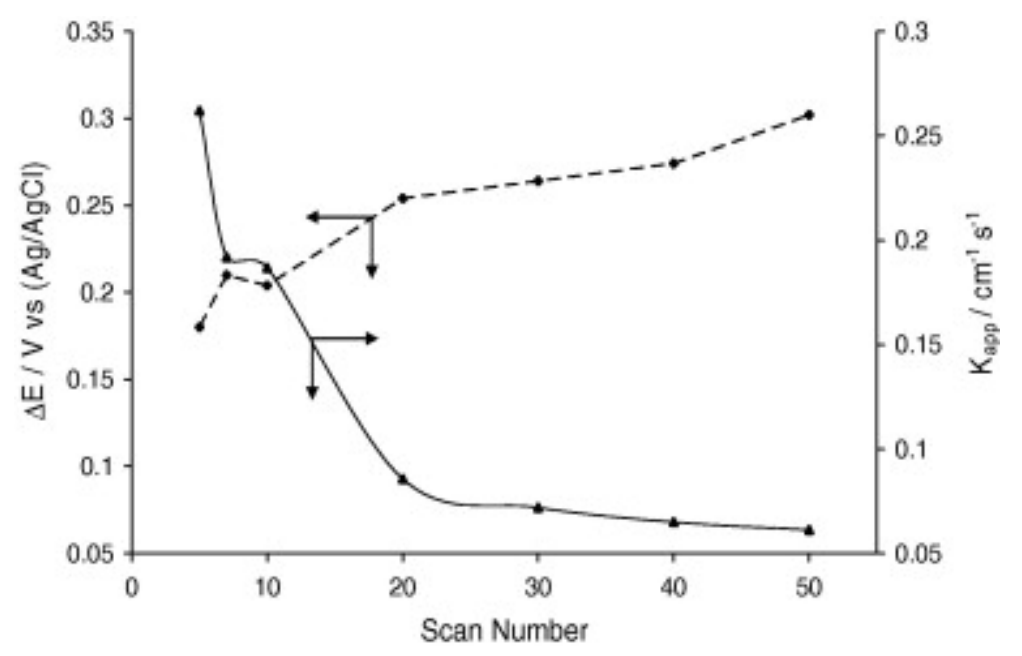

Fig. 2. Plots of the peak-to-peak separation $\left(\Delta E_{\mathrm{p}}\right)$ and apparent electron transfer constant $\left(k_{\text {app }}\right)$ versus scan number.

It is evident from Fig. 2 that low electropolymerization scans $(<10)$ gave faster electron transfer kinetics compared to scan numbers greater than 10, meaning that at the conditions employed in this work the best electrode response was observed at low scan numbers of NiPc electropolymers. This result also shows the possibility to control the amount of polymeric species that can be deposited onto the electrode at constant concentration of the MPc.

\subsection{Comparative electron transport properties}

Fig. 3 presents the comparative typical CVs (Fig. 3a) and their corresponding Nyquist plots (Fig. 3b) for the BPPGE-SWCNT-poly-NiTAPc 7 and other electrodes. 
a
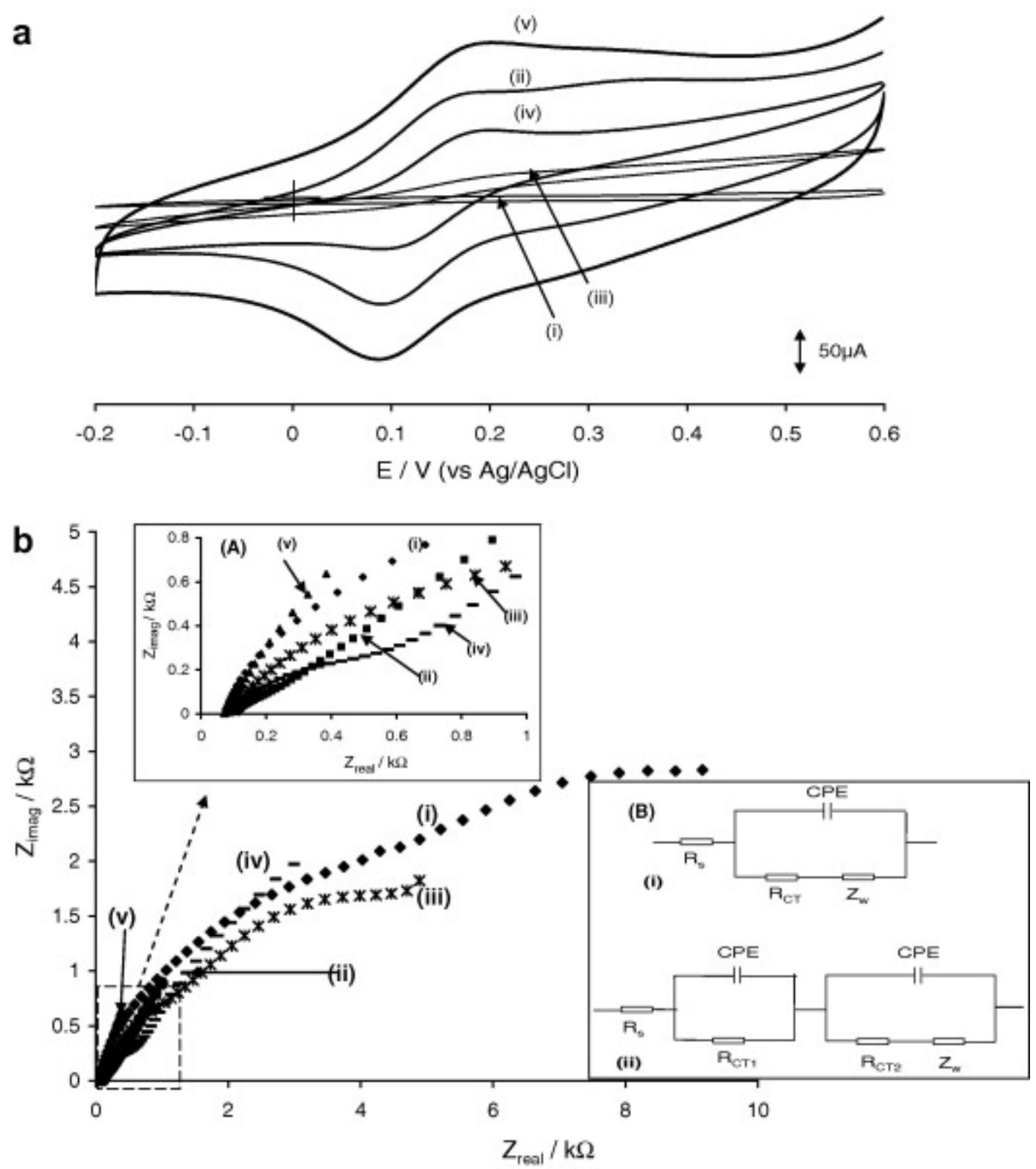

Fig. 3. (a) Cyclic voltammetric profiles and (b) Nyquist plots of plots resulting from bare BPPGE (i), BPPGE-poly-NiTAPc 7 (ii), BPPGE-poly-Ni(OH)TAPc 7 (iii), BPPGESWCNT (iv) and BPPGE-SWCNT-poly-NiTAPC 7 (v) in $0.1 \mathrm{M} \mathrm{KCl}$ containing equimolar mixture of $\mathrm{K}_{4} \mathrm{Fe}(\mathrm{CN})_{6}$ and $\mathrm{K}_{3} \mathrm{Fe}(\mathrm{CN})_{6}$. Scan rate for $\mathrm{CV}=50 \mathrm{mV} / \mathrm{s}$. Inset $\mathrm{A}$ of (b) is the expanded part of the high frequency region, while inset B is the equivalent circuits used to fit the EIS data of the electrodes. 
From the Nyquist plots, BPPGE and BPPGE-poly-Ni(OH)TAPc ${ }_{7}$ gave two depressed circles with Warburg lines, the other electrodes exhibited essentially one semi-circle with a Warburg line. The most widely accepted explanation for the appearance of depressed circles in Nyquist plots is the occurrence of different electrochemical processes that take place at different RC time constants [28]. Such processes arise because of microscopic roughness or high porosity of the electrodes causing an inhomogeneous distribution of the solution resistance and in the double layer capacitance. Based on the results, the following equivalent circuits (Fig. 3, inset B) were chosen for fitting the impedance plots of the BPPGE (Fig. 3, inset B(i)), BPPGE-poly-Ni(OH)TAPc 7 (Fig. 3 inset B(ii)), and BPPGE-SWCNT-poly-NiTAPc 7 , BPPGE-SWCNT and BPPGE-poly-NiTAPc (Fig. 3, inset B(i)). Here, $R_{\mathrm{s}}$ is the solution/electrolyte resistance, $Q$ is the capacitance of the film/electrolyte interface of the constant phase element, $\mathrm{CPE}, R_{\mathrm{CT}}$ represents the chargetransfer resistance at the electrode/electrolyte interface, and $Z_{\mathrm{w}}$ is the Warburg impedance related to the diffusion of the ions in the bulk electrode. EIS data are summarized in Table 1. At the experimental conditions employed in this work, it is clear that electron transport is much faster at the BPPGE-SWCNT-poly-NiTAPc ${ }_{7}$ and slowest at the BPPGE-poly-Ni(OH)TAPc${ }_{7}$. 
Table 1.

Comparative EIS data obtained for the electrodes

\begin{tabular}{|c|c|c|c|c|c|c|}
\hline \multirow[t]{2}{*}{ Electrode } & \multicolumn{6}{|c|}{ Electrochemical impedance spectral parameter ${ }^{a}$} \\
\hline & $R_{\mathrm{s}}\left(\Omega \mathrm{cm}^{2}\right)$ & $\mathrm{CPE}\left(\mu \mathrm{F} \mathrm{cm}^{-2}\right)$ & $n$ & $R_{\mathrm{CT}}\left(\mathrm{k} \Omega \mathrm{cm}^{2}\right)$ & $10^{6} Z_{\mathrm{w}}\left(\Omega \mathrm{cm}^{2} \mathrm{~s}^{-1 / 2}\right)$ & $10^{4} k_{\text {app }}\left(\mathrm{cm}^{-2} \mathrm{~s}^{-1}\right)$ \\
\hline BPPGE & $10.05(7.6)$ & $0.51(17.3)$ & $0.71(1.9)$ & $0.864(5.8)$ & $36.4(8.0)$ & $3.1 \pm 0.2$ \\
\hline BPPGE-poly-NiTAPc & $34.90(0.2)$ & $229.00(3.7)$ & $0.87(0.6)$ & $0.135(2.3)$ & $186.4(1.2)$ & $19.7 \pm 0.5$ \\
\hline BPPGE-poly-Ni(OH)TAPc & $17.21(1.1)$ & $11.58(\approx 30)$ & $0.71(3.2)$ & $1.097(\sim 10)$ & $215.3(1.4)$ & $2.4 \pm 2.4$ \\
\hline BPPGE-SWCNT & $13.25(3.4)$ & $0.72(\approx 30)$ & $0.61(3.6)$ & $0.130(10.6)$ & $61.4(3.3)$ & $20.5 \pm 2.2$ \\
\hline BPPGE-SWCNT-poly-NiTAPc 7 & $14.03(1.0)$ & $10.51(9.4)$ & $0.74(1.4)$ & $0.101(3.7)$ & $126.8(1.6)$ & $26.4 \pm 1.0$ \\
\hline
\end{tabular}

${ }^{\mathrm{a}}$ The values in brackets are the estimated errors in percent obtained by fitting the experimental data with the model (see inset, Fig. $3 b)$.

${ }^{\mathrm{b}}$ Unlike the other electrodes, data were fitted with the equivalent circuit (Fig. 3b, inset B(ii)).

The impedance of the CPE $\left(Z_{C P E}\right)$ is a power-law dependent interfacial capacity given as [28] and [29] 


$$
Z_{\mathrm{CPE}}=\left[Q(j \omega)^{n}\right]^{-1}
$$

where $Q$ is the frequency-independent constant, $\omega$ is the radial frequency, and $n$ is an exponent related to the depression angle. An $n$ value of zero corresponds to a pure resistor; a unit value of $n$ corresponds to a pure capacitor while a 0.5 value corresponds to Warburg impedance. Our choice of the CPE model for fitting the EIS data is because it represents the real world model that takes cognizance of topological imperfections of the electrode surface caused by the crystal facets and the inherent roughness of the BPPGE. As can be seen, the EIS data satisfactorily fitted the chosen equivalent circuits. The high estimated error percentage for the CPE value (17-30\%) observed in the fitting of the BPPGE, BPPGE-poly-Ni(OH)TAPc and BPPGE-SWCNT is the consequence of the roughness and porosity of these electrodes. As we expected for the CPE model, $n$ values were approximately in the $0.6-0.9$ range, confirming that the electrodes behave like pseudocapacitors.

Also, the impact of solution $\mathrm{pH}$ on the electron transport kinetics of the BPPGE$\mathrm{SWCNT}_{\text {-poly-NiTAPc}}$ at different $\mathrm{pHs}$ of a $1 \mathrm{mM}$ of $\mathrm{K}_{4} \mathrm{Fe}(\mathrm{CN})_{6} / \mathrm{K}_{3} \mathrm{Fe}(\mathrm{CN})_{6}$ solution was investigated. The $\Delta E_{\mathrm{p}}$ and $R_{\mathrm{CT}}$ values increased with increasing $\mathrm{pH}$, indicating consistent decrease in electron transfer kinetics with increase in $\mathrm{pH}$. This behaviour suggests that increasing deprotonation of the crystalline electropolymeric film leads to increasing inhibition of the anionic redox probe from penetrating into the electrode surface, hence the loss of Faradaic response.

\subsection{Comparative film capacitances}

Comparative CVs of the electrodes at different $\mathrm{pH}$ of buffer solutions (0.1 M PBS, $\mathrm{pH}$ 3.0-10.0) revealed that the cathodic and anodic charges associated with the BPPGESWCNT-poly-NiTAPc 7 are much higher than the charges obtained at the BPPGESWCNT, BPPGE-poly-NiTAPc ${ }_{7}$ or BPPGE-poly-Ni(OH)TAPc ${ }_{7}$ electrodes (exemplified in Fig. 4 for $\mathrm{pH}$ 3.0). 


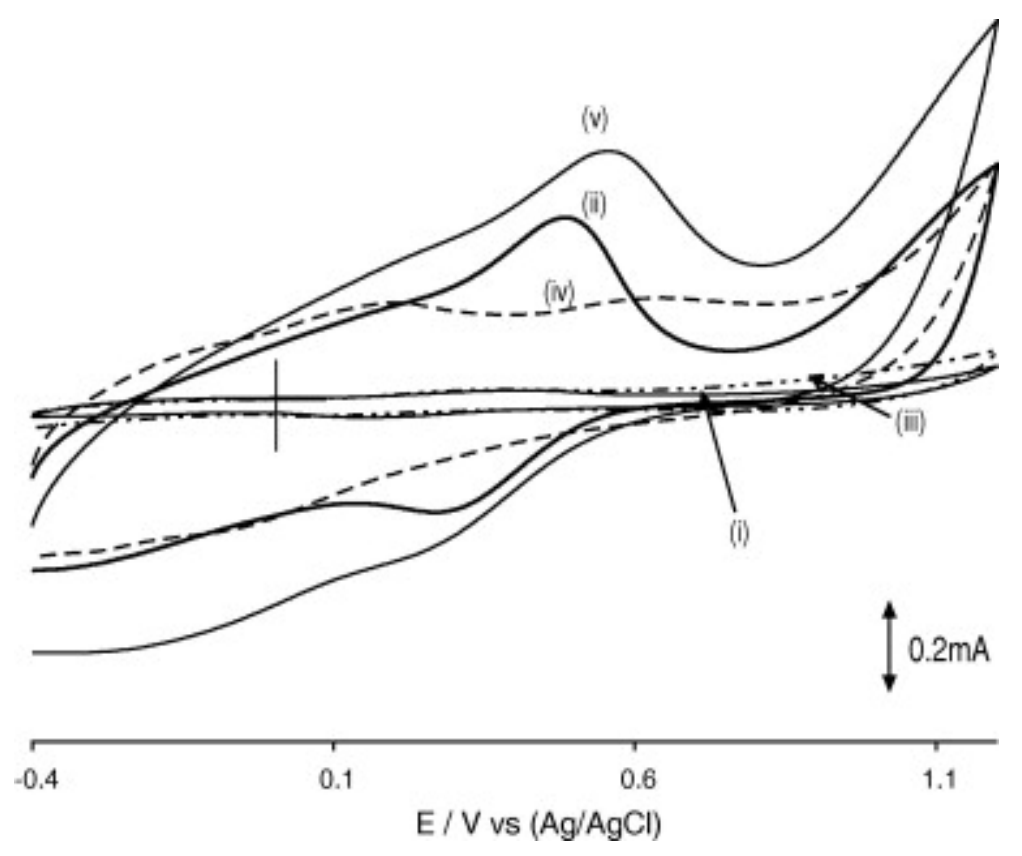

Fig. 4. Cyclic voltammograms of the bare BPPGE (i), BPPGE-poly-NiTAPc 7 (ii), BPPGE-poly-Ni(OH)TAPc 7 (iii), BPPGE-SWCNT (iv) and BPPGE-SWCNT-poly$\mathrm{NiTAPC}_{7}(\mathrm{v})$ in $0.1 \mathrm{M}$ PBS (pH 3.0).

For example, at pH 3.0, the estimated anodic charges obtained for BPPGE (i), BPPGESWCNT (ii), BPPGE-poly-NiTAPc 7 (iii), BPPGE-poly-Ni(OH)TAPc 7 (iv) and BPPGESWCNT-poly-NiTAPc 7 (v) are $7.2 \times 10^{-4}, 5.7 \times 10^{-3}, 1.1 \times 10^{-2}, 7.6 \times 10^{-4}$ and $1.4 \times 10^{-2} \mathrm{C} \mathrm{cm}^{-2}$, respectively. The values obtained for the respective electrodes at neutral and alkaline $\mathrm{pH}$ conditions were in the same magnitude as for the acidic conditions. This result is consistent with organized electrocrystals, for example, a recent report by López and Choi [21] where zinc crystals grown attached to three dimensionally dispersed dendrite backbones efficiently increased interfacial areas and were found to have much higher charges than the corresponding dendritic zinc films of irregular features or electrodes composed with individual crystals. Insight into the ability of the films to store charges may be obtained from the film capacitance $\left(C_{\text {filmm }} / \mathrm{F} \mathrm{cm}^{-2}\right)$ estimated in the non-Faradaic regimes (0.93 V versus $\mathrm{Ag} \mid \mathrm{AgCl})$ using the simple expression [30]: 


$$
C_{1+m}=\frac{l_{\mathrm{ch}}}{\mathrm{s} A}
$$

where $I_{\mathrm{ch}}$ is the charging current, $v$ the scan rate and $A$ the geometric area of the electrode. The capacitance at the $\mathrm{pH} 3.0$ was estimated to be $1.1 \times 10^{-2} \mathrm{~F} \mathrm{~cm}^{-2}$ for the BPPGESWCNT-poly-NiTAPc 7 compared to the $7.7 \times 10^{-3}, 5.5 \times 10^{-3}$ and $1.8 \times 10^{-3} \mathrm{~F} \mathrm{~cm}^{-2}$ for the BPPGE-SWCNT, BPPGE-poly-NiTAPc ${ }_{7}$ and the BPPGE-poly-Ni(OH)TAPc 7 , respectively. In the $\mathrm{pH} 7.2$ conditions, the value for the BPPGE-SWCNT-poly-NiTAPc 7 was $4.2 \times 10^{-3} \mathrm{~F} \mathrm{~cm}^{-2}$ compared to BPPGE-poly-NiTAPc $7\left(1.7 \times 10^{-3} \mathrm{~F} \mathrm{~cm}^{-2}\right)$ and BPPGE-poly-Ni(OH)TAPc $7\left(3.2 \times 10^{-3} \mathrm{~F} \mathrm{~cm}^{-2}\right)$ electrodes without SWCNT. Interestingly, despite the significant decrease $(c a .62 \%)$ in the capacitance of the BPPGESWCNT-poly-NiTAPc 7 from acidic to the neutral $\mathrm{pH}$, the capacitance of the BPPGESWCNT-poly-NiTAPc 7 is still significantly greater (ca. 27-50\%) than those of the electrode without SWCNT, i.e., BPPGE-poly-NiTAPc 7 and the BPPGE-poly$\mathrm{Ni}(\mathrm{OH}) \mathrm{TAPc}_{7}$.

\subsection{Comparative electrocatalytic behaviour towards nitric oxide}

Electropolymeric films of NiPc complexes are popular for the electrocatalytic detection of nitric oxide (NO) as reviewed elsewhere [6]. The NO was generated from sodium nitrite following the established literature reports [31]. It is known that in acid solution $(\mathrm{pH}<4) \mathrm{NaNO}_{2}$ generates mainly free $\mathrm{NO}$ by disproportionation. Fig. 5 presents comparative square wave voltammograms for the NO using the different electrodes. 


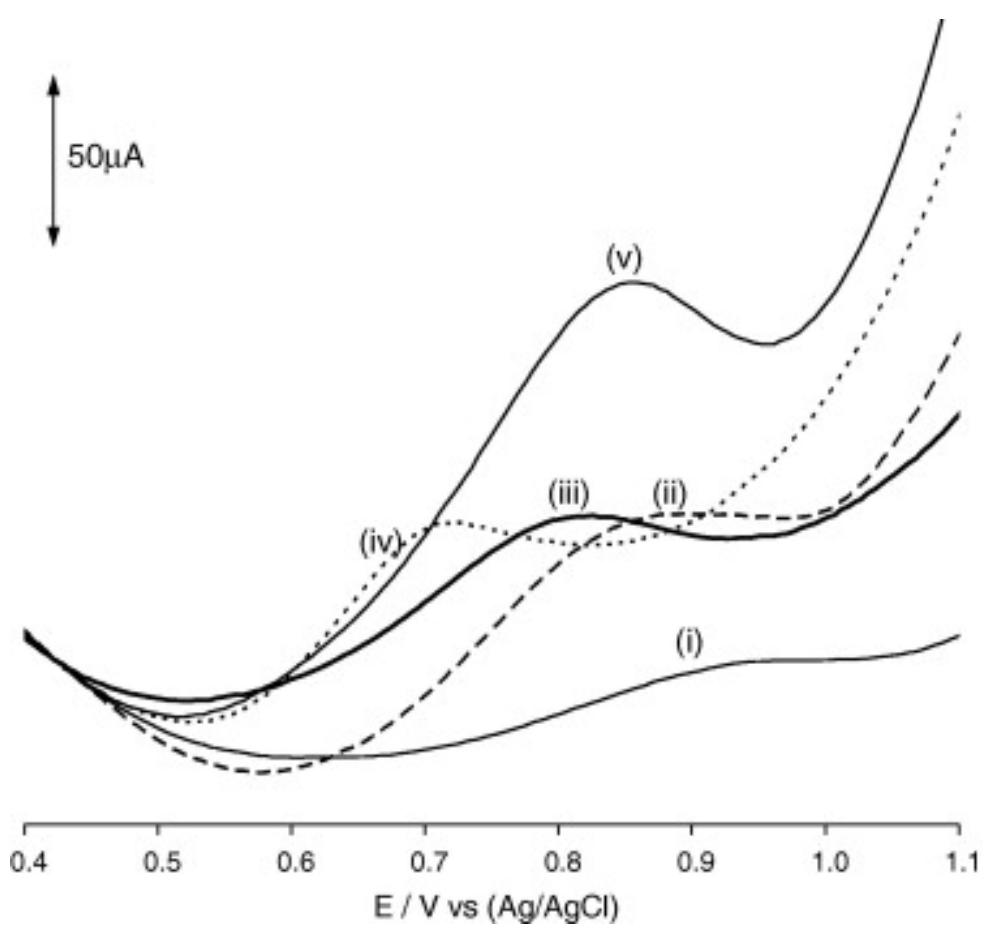

Fig. 5. SWV illustrating the responses in (a) $10^{-4} \mathrm{M}$ NO of the bare BPPGE (i), BPPGEpoly-NiTAPc 7 (ii), BPPGE-poly-Ni(OH)TAPc 7 (iii), BPPGE-SWCNT (iv) and BPPGESWCNT-poly-NiTAPC 7 (v).

The catalytic currents follow the trend: BPPGE-SWCNT-poly-NiTAPc 7 BPPGE-poly$\mathrm{Ni}(\mathrm{OH}) \mathrm{TAPc}_{7}>\mathrm{BPPGE} \mathrm{BiTAPc}_{7}>\mathrm{BPPGE}_{\mathrm{SWCNT}}>$ BPPGE . From the catalytic current responses, it can be implied that SWCNT, by inducing crystallinity onto NiTAPc electropolymeric film, is able to enhance the electronic communication between polyNiTAPc and the BPPGE.

\section{Conclusion}

In this work, we report that SWCNT-confined on basal plane pyrolytic graphite electrode induces crystallinity on the NiTAPc electropolymeric film and significantly affects the redox properties of the NiTAPc film. The results clearly indicate some potential advantages of using the electrode based on the SWCNT-NiTAPc hybrid for catalytic and sensing applications as the electrode showed about two times higher catalytic current for the detection of nitric oxide compared to bare BPPGE, BPPGE-SWCNT and other electrodes without SWCNTs. 


\section{References}

[1] C.P. Poole and F.J. Owens, Introduction to Nanotechnology, Wiley Interscience, Hoboken, NJ (2003) (Chapter 5, p. 103).

[2] C.E. Banks, R.R. Moore, T.J. Davies and R.G. Compton, Chem. Commun. (2004), p. 1804.

[3] R.R. Moore, C.E. Banks and R.G. Compton, Anal. Chem. 76 (2004), p. 2677.

[4] In: K.M. Kadish, K.M. Smith and R. Guilard, Editors, The Porphyrin Handbook vols. 15-20, Academic Press, Boston (2003) (Chapters 97-122).

[5] In: C.C. Leznoff and A.B.P. Lever, Editors, Phthalocyanines: Properties and Applications vols. 1-4, VCH Publishers, New York $(1989,1996)$.

[6] K.I. Ozoemena and T. Nyokong In: C. A Grimes, E.C. Dickey and M.V. Pishko, Editors, Encyclopedia of Sensors vol. 3, American Scientific Publishers, California (2006), p. 157 (Chapter E).

[7] M.P. Siswana, K.I. Ozoemena and T. Nyokong, Electrochim. Acta 52 (2006), p. 14.

[8] K.I. Ozoemena, J. Pillay and T. Nyokong, Electrochem. Commun. 8 (2006), p. 1391.

[9] J. Pillay and K.I. Ozoemena, Electrochim. Acta 52 (2007), p. 630.

[10] D.M. Guldi, H. Taieb, G.M.A. Rahman, N. Tagmatarchis and M. Prato, Adv. Mater. 17 (2005), p. 871.

[11] G.M.A. Rahman, D.M. Guldi, S. Campidelli and M. Prato, J. Mater. Chem. 16 (2006), p. 62.

[12] S.-N. Li, Z.-M. Li, M.-B. Yang, Z.-Q. Hu, X.-B. Xu and R. Huang, Mater. Lett. 58 (2004), p. 3967.

[13] S. Tzavalas, V. Drakonakis, D.E. Mouzakis, D. Fischer and V.G. Gregoriou, Macromolecules 39 (2006), p. 150.

[14] J.Y. Kim, H.S. Park and S.H. Kim, J. Appl. Polym. Sci. 103 (2007), p. 1450.

[15] T.-M. Wu and E.-C. Chen, Polym. Eng. Sci. 46 (2006), p. 1309.

[16] L. Li, C.Y. Li and C. Ni, J. Am. Chem. Soc. 128 (2006), p. 692.

[17] B.P. Grady, F. Pompeo, R.L. Shambaugh and D.E. Resasco, J. Phys. Chem. B 106 (2002), p. 5852.

[18] J. Geng and T. Zeng, J. Am. Chem. Soc. 128 (2006), p. 6827. 
[19] S.M. Keogh, T.G. Hedderman, M.G. Ruther, F.M. Lyng, E. Gregan, G.F. Farrell, G. Chambers and H.J. Byrne, J. Phys. Chem. B 109 (2005), p. 5600.

[20] P. Batail, K. Boubekeur, M. Fourmigue and J.-C.P. Gabriel, Chem. Mater. 10 (1998), p. 3005.

[21] C.M. López and K.-S. Choi, Langmuir 22 (2006), p. 0625.

[22] T. Nyokong and S. Vilakazi, Talanta 61 (2003), p. 27.

[23] Z.H. Taha, Talanta 61 (2003), p. 3.

[24] C. Wartelle, N.P. Rodrigues, M. Koudelka-Hep and F. Bedioui, Mater. Sci. Eng.: C 26 (2006), p. 534.

[25] M. Biesaga, K. Pyrzynska and M. Trojanowicz, Talanta 51 (2000), p. 209.

[26] J. Liu, A.G. Rinzler, H. Dai, J.H. Hanfer, R.K. Bradley, P.J. Boul, A. Lu, T. Iverson, K. Shelimov, C.B. Huffman, F.R. Macias, Y.S. Shon, T.R. Lee, D.T. Colbert and R.E. Smalley, Science 280 (1998), p. 253.

[27] E. Katz and I. Wilner In: V.M. Mirsky, Editor, Ultrathin Electrochemical Chemoand Biosensors. Technology and Performance, Springer Verlag, New York (2004), p. 68 (Chapter 4).

[28] In: E. Barsoukov and J.R. Macdonald, Editors, Impedance Spectroscopy: Theory

Experiment and Applications (second edn.), Wiley, Hoboken, NJ (2005) (Chapters 1-4).

[29] L.K. Xu and J.D. Scantlebury, J. Electrochem. Soc. 150 (2003), p. B254.

[30] J. Jiang and A. Kucernak, Synth. Met. 114 (2000), p. 209.

[31] A. Yu, Z. Liang, J. Cho and F. Caruso, Nano Lett. 3 (2003), p. 1203. 